\title{
Performance Evaluation of a High-field Orbitrap Mass Analyzer
}

\author{
Alexander Makarov, Eduard Denisov, and Oliver Lange \\ Thermo Fisher Scientific, Bremen, Germany
}

A new design of the Orbitrap ${ }^{\mathrm{TM}}$ mass analyzer is presented. Higher frequencies of ion oscillations and hence higher resolving power over fixed acquisition time are achieved by decreasing the gap between the inner and outer Orbitrap electrodes, thus providing higher field strength for a given voltage. Experimental results confirm maximum FWHM resolving power in excess of 350,000 at $\mathrm{m} / \mathrm{z} 524$ and 600,000 at $\mathrm{m} / \mathrm{z} 195$, isotopic resolution of proteins above $40 \mathrm{kDa}$, and a single-shot dynamic range of 25,000. It was also found that mass shifts in the new design depend very little on space charge inside the analyzer. This performance was achieved using higher voltages and by careful balancing of construction tolerances and operation parameters, which appeared to vary in narrower ranges of tuning than for a standard Orbitrap analyzer. (J Am Soc Mass Spectrom 2009, 20, 1391-1396) (C 2009 Published by Elsevier Inc. on behalf of American Society for Mass Spectrometry

A 11 trapping mass analyzers are known to benefit from increasing strength of the trapping field, major benefits being higher dynamic range, repetition rate, resolving power, tolerance to space charge, etc. These benefits have become the major driver in the quest for higher and higher field magnets in Fourier transform ion cyclotron resonance (FT ICR) mass spectrometry [1, 2]. Similar improvements could be naturally expected for another prominent member of Fourier transform mass spectrometry family; the Orbi$\operatorname{trap}^{\mathrm{TM}}$ mass analyzer [3, 4]. The electrostatic nature of trapping in this analyzer determines the difference in strategies. In FT ICR the field strength could be increased only by changing the magnet to a more powerful one, the latter becoming the most expensive part of the mass spectrometer, while in an electrostatic trap it could be increased not only by applying higher voltages but also by changing geometry of the trap.

This work investigates, both theoretically and experimentally, the performance of the Orbitrap mass analyzer with an optimized field structure, including a design intended to provide increased field strength at a given voltage.

\section{Theoretical}

Typically, the Orbitrap mass analyzer consists of an outer barrel-like electrode of maximum radius $R_{2}$ and a central spindle-like electrode along the axis of radius $R_{1}$, with the outer electrode maintained at virtual ground of preamplifier and the central electrode at a voltage, $-U r$

Address reprint requests to Dr. A. Makarov, Thermo Fisher Scientific (Bremen) GmbH, Hanna-Kunath-Str. 11, 28199 Bremen, Germany. E-mail: alexander.makarov@thermofisher.com
(Ur $>0$ for positive ions) [3, 4]. Thus, the general equation for the quadro-logarithmic potential distribution between the electrodes, $U(r, z)[3]$, can be re-written in the form:

$$
U(r, z)=\frac{k}{2}\left(z^{2}-\frac{\left(r^{2}-R_{1}^{2}\right)}{2}\right)+\frac{k}{2} \cdot R_{m}^{2} \cdot \ln \left[\frac{r}{R_{1}}\right]-U r
$$

where $r, z$ are cylindrical coordinates $(z=0$ being the plane of the symmetry of the field), $k$ is the field curvature, and $R_{m}$ is the characteristic radius that corresponds to radius at which $d U(r, z) /\left.d r\right|_{z=0}=0$, i.e., the field stops attracting stationary ions towards the axis and starts to repel them (typically, $R_{m}>R_{2} \sqrt{2}$ during electrostatic trapping [3]). Boundary condition $U\left(R_{2}, 0\right)=0$ allows one to determine the field curvature $k$, which in its turn defines the frequency of axial oscillations:

$$
\omega=\sqrt{\frac{e}{(m / z)} \cdot k}
$$

where $e$ is the elementary charge $\left(1.602 \times 10^{-19} \mathrm{C}\right)$. The latter can be re-written as:

$$
\omega=\sqrt{\frac{e}{(m / z)} \cdot \frac{2 \cdot U r}{R_{m}^{2} \ln \left(\frac{R_{2}}{R_{1}}\right)-\frac{1}{2}\left[R_{2}^{2}-R_{1}^{2}\right]}}
$$

This equation shows that the frequency increases proportionally to the square root of applied voltage $U r$ and inversely proportionally to the scaling of the trap (i.e., simultaneous increase of $R_{m}, R_{1}, R_{2}$ ). As the frequency increases, so do other important parameters: the maxi- 
mum resolving power and dynamic range over a fixed duration of acquisition, acquisition speed at a fixed resolving power, tolerance to space charge.

Thus, this equation reveals the most straightforward ways of improving Orbitrap performance: to increase voltage on the central electrode and scale the trap down. Unfortunately, straightforward solutions are not always the best. For example, increase of voltage provides relatively low "return on investment" because of square-root dependence, whereas associated problems such as power dissipation, probability of breakdown, and required dimensions of isolators increase linearly or quadratically with voltage. Similarly, geometrical scaling-down of the trap requires also scaling-down of absolute manufacturing tolerances and the injection slot to keep relative field perturbations under control. The former requires revision of manufacturing processes while the latter demands drastic changes of the injection optics to avoid loss of sensitivity because of quadratic scaling-down of the slot cross-section.

Another way to increase axial frequency in (eq 3) could be the change of ratios between $R_{m}, R_{1}$, and $R_{2}$. This approach could also be combined with any of the previously described approaches. In a standard Orbitrap analyzer, $R_{1}=6 \mathrm{~mm}$ and $R_{2}=15 \mathrm{~mm}$, i.e., $\ln \left(R_{2} / R_{1}\right)=0.916$, which makes the first term in the denominator in ( eq 3 ) dominating. This ratio becomes $\ln \left(R_{2} / R_{1}\right)=0.51$ for $R_{1}=9 \mathrm{~mm}$ and unchanged $R_{m}$ and $R_{2}$ in the new design. Presence of the negative second term only enhances this change which results in frequency increase by a factor of 1.4, almost proportionally to the factor of 1.5 change in $R_{1}$. Frequency also could be increased by reducing the characteristic radius $R_{m}$. However it has already been reduced in a standard Orbitrap analyzer close to the recommended minimum $R_{2} \sqrt{2}$.

Simulation software that has been developed inhouse and standard analytical models have been employed for ion-optical design and evaluation of Orbitrap electrodes with unchanged outer electrodes and a new central electrode. A new maximum radius of the central electrode $(9 \mathrm{~mm}$ ) was chosen to be as close as possible to ion trajectories but still far enough to minimize the influence of manufacturing imperfections (Figure 1). In addition, voltage on the central electrode during detection has been increased from the standard setting of $3.5 \mathrm{kV}$ to $5 \mathrm{kV}$, which results in the total frequency increase by a factor of $F=1.7$. Similarly to high-field Fourier transform ion cyclotron resonance (FT ICR) instruments, the new design has been named high-field Orbitrap (HF Orbitrap).

Increase of the field by this approach also results in certain adverse effects. One of them is the reduction of the image current on the outer electrodes as the larger central electrode absorbs a significantly higher proportion of the image current due to its closer proximity to ion trajectories. This effect was partially compensated by increasing the gap between the split outer electrodes
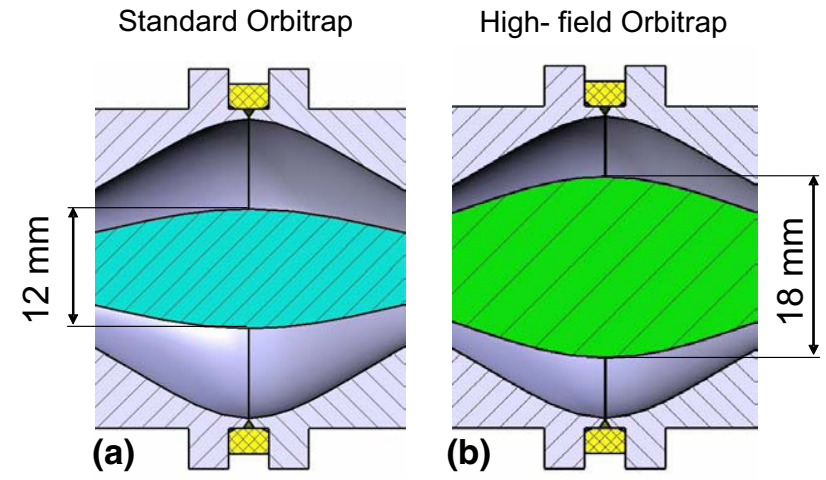

Figure 1. Comparison of a standard Orbitrap geometry (a) with that of a high-field Orbitrap analyzer (b). Shape of outer electrodes is identical for both cases, largest i.d. of outer electrodes being $30 \mathrm{~mm}$.

by a factor of three to reduce overall capacitance of detection circuitry and improve signal-to-noise ratio.

Another adverse effect of a thicker central electrode appeared to be reduced pumping of the trapping volume. This effect was counteracted by making larger pumping apertures on the ends of the outer electrodes. As this change took place on the periphery of the trapping field, its effect on ion-optical parameters was calculated to be negligible.

One more effect, increase of the required initial ion energy, comes directly from the increase of the field strength. For an ion to move circularly at radius $R$ during detection, equilibrium tangential velocity $v_{\varphi}$ should be [3]

$$
\frac{(m / z) \cdot v_{\varphi}^{2}}{2 e}=\frac{k}{4} \cdot\left(R_{m}^{2}-R^{2}\right)
$$

Following off-axis injection in the Orbitrap mass analyzer [3] at radius $R_{i}$, ions are subjected to electrodynamic squeezing by increasing voltage on the central electrode. Due to conservation of angular momentum ion acceleration voltage $V$ could be linked to tangential velocity $v_{\varphi}$ via injection radius $R_{i}$ and final radius $R$ :

$$
R \cdot v_{\varphi}=R_{i} \sqrt{\frac{2 \cdot e V}{(m / z)}}
$$

As a result, ions need to be accelerated before entering the trap by voltage.

$$
V=\frac{k}{4} \cdot\left(R_{m}^{2}-R^{2}\right) \cdot\left(\frac{R}{R_{i}}\right)^{2}
$$

When comparing a high-field Orbitrap analyzer (index $\mathrm{HF}$ ) and a standard one (index ST) for increase of frequency (eqs 2,3) by factor $F$ at constant $R_{m}$ and injection radius $R_{i}$, the acceleration voltage must increase as 


$$
\frac{V_{H F}}{V_{S T}} \approx F^{2} \cdot\left(\frac{R_{H F}}{R_{S T}}\right)^{2}
$$

wherein $R_{H F}$ and $R_{S T}$ are final equilibrium radii in high-field and standard Orbitrap analyzer, respectively. Typically, $R_{H F}=10 \ldots 10.5 \mathrm{~mm}, R_{S T}=9.10 \mathrm{~mm}$, which for $F=1.7$ results in $\left(V_{H F} / V_{S T}\right)=3 \ldots 3.5$. This increase is so great that it has required the re-design of existing electronics driving the C-trap [4]. It also means that center-of-mass collision energy of ions with background gas scales up almost as fast as the acceleration voltage (actually, as $F^{2}$ ). Consequences of this feature are discussed below in relation to Figure 5 .

\section{Experimental}

Using an LTQ ${ }^{\mathrm{TM}}$ Orbitrap XL mass spectrometer [4] as a test-bed and modified electronics, a standard commercial Orbitrap mass analyzer has been compared with a new design, featuring a thicker central electrode and the same outer electrodes (Figure 1). To improve the ultimate vacuum, higher power bake-out heaters were employed to raise bake-out temperature from the standard $110^{\circ} \mathrm{C}$ to $180^{\circ} \mathrm{C}$. This allowed pressure to go below $10^{-10}$ mbar level following just 12-h bake-out after venting. Overheating of the preamplifier linked to the chamber was avoided by water-cooling of its box.

Experiments were aimed to determine analyzer characteristics such as maximum resolution, resolution per detect time, dynamic range, and tolerance to space charge. Frequency shifts caused by space charge have been compared with experimental results from a standard LTQ Orbitrap XL instrument (with $3.5 \mathrm{kV}$ central electrode voltage), 12 Tesla LTQ FT hybrid mass spectrometer with Ultra cell from University of Illinois [5], as well as from a 14.5 Tesla Linear trap/FTICR instrument with a cylindrical ICR cell from National High Magnetic Field Laboratory (NHMFL) [6]. Due to differences in transfer efficiency, excitation amplitude, detection circuitry, and processing software, direct compar-

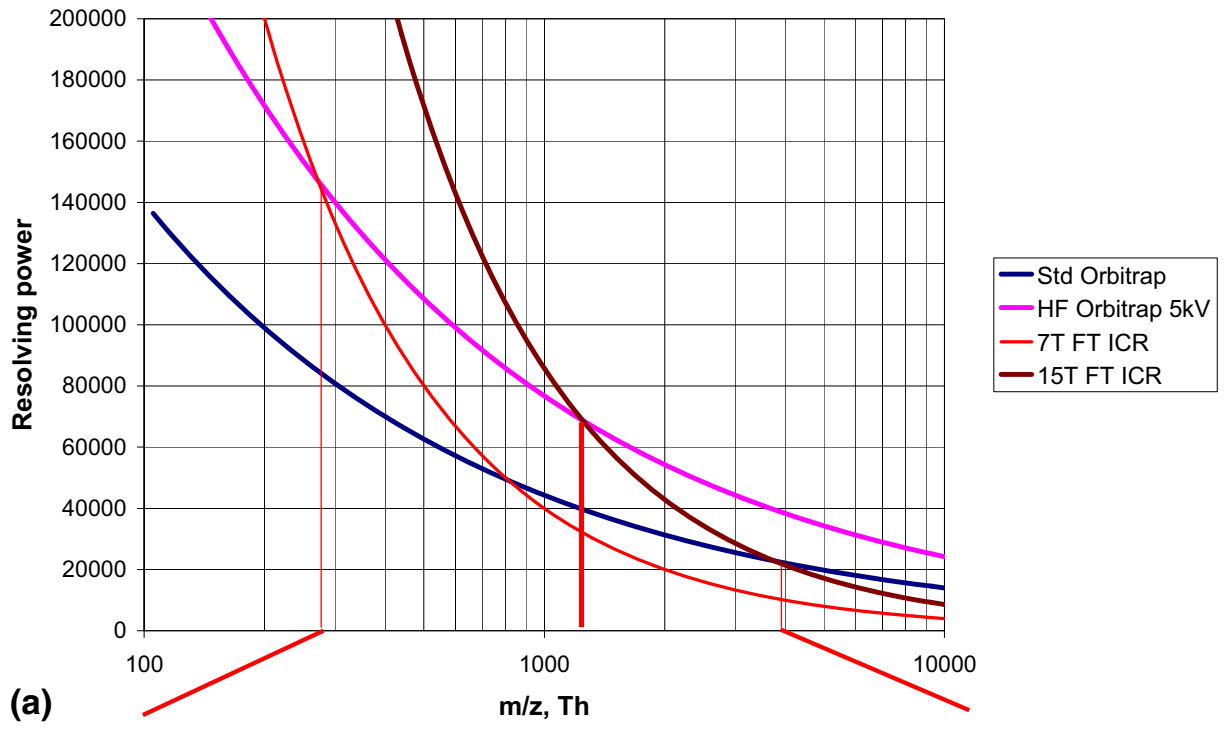

(a)

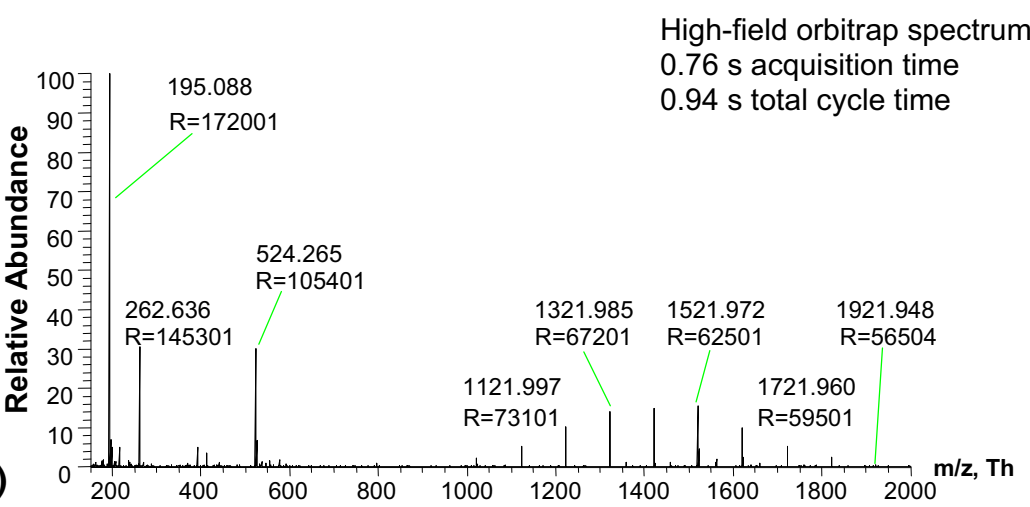

Figure 2. (a) Resolving power as a function of $m / z$ for different FTMS instruments: Standard Orbitrap analyzer [4]; high-field Orbitrap analyzer; FT ICR with 7 Tesla superconducting magnet; FT ICR with 15 Tesla superconducting magnet. Acquisition parameters are the same in all cases $(0.76 \mathrm{~s}$ detection time, single zero-filling and Kaiser-Bessel apodization). (b) An example of a spectrum with the mass range $m / z$ 150-2000 acquired using HF Orbitrap. 
ison of detected ion numbers in these instruments appeared not to be straightforward. As all of these instruments share the same linear trap (LT) front-end [7] with the same calibration routine, it was the target value of the linear trap that was used as a basis for estimating ion load. Target value of the LT is closely related to the actual number of ions stored in it.

Experiments have been carried out using the standard LTQ calibration mixture with caffeine, the peptide MRFA, and Ultramark 1600 dissolved in 25:25:49:1 vol.vol.vol water/methanol/acetonitrile/acetic acid solution. Yeast enolase protein was obtained from SigmaAldrich Chemie GmbH (Munich, Germany) and used without further purification.

\section{Results and Discussion}

As expected, increased field strength inside the new Orbitrap mass analyzer has allowed to significantly increase resolving power at a given acquisition time by about $50 \%$ at the same voltage, and by $80 \%$ at an increased voltage on the central electrode (Figure 2). An experimental resolving power of around 120,000 (at $\mathrm{m} / \mathrm{z}$ 400) has been demonstrated at $1 \mathrm{scan} / \mathrm{s}$, which is $20 \%$ better than on a commercially available 7T FT-ICR, but still less than the theoretical limit for $12 \mathrm{~T}$ or $15 \mathrm{~T}$ FT-ICR. It should be noted that due to the different dependencies of resolving power on mass for the different analyzer types (Orbitrap versus FT-ICR), the new Orbitrap geometry provides higher frequency and hence higher resolving power comparing to $7 \mathrm{~T}$ and $15 \mathrm{~T}$ FT-ICR for a fixed scan duration at masses above $280 \mathrm{u}$ and $1300 \mathrm{u}$, respectively (Figure 2).
It was noticed that the reduced gap between the central and the outer electrodes of the Orbitrap increases requirements on the quality of alignment and tuning in the preceding C-trap and ion optics. On the other hand, increase of acceleration voltage in ion optics by a factor of about 3 (up to $3.5 \mathrm{kV}$ ) improves extraction and transport of ions. This also offsets the decrease of detection efficiency due to the increased shielding of induced image current by the central electrode. As a result, the sensitivity of the HF Orbitrap is comparable to that of a standard Orbitrap. Experiments on maximum dynamic range in a single shot (Figure 3) also support this statement. Using the multi-fill approach of the C-trap [8], a controlled mixture of ions with very high abundance differences has been created, and the dynamic range in a single $0.76 \mathrm{~s}$ acquisition proved to exceed 25,000 comparing peaks with S/N 50,000 and S/N 2. Total target value T for the linear trap has been $\mathrm{T}=10^{7}$, i.e., the C-trap was filled up to its complete saturation.

Figure 4 shows apparent shifts of frequency in four different FTMS instruments relative to measured frequency at a low ion load (a linear trap target value of 10,000 for Orbitraps and 50,000 for FT ICR). FT ICR mass analyzer with a grid (Ultra) cell [9] has shown a markedly different trend of frequency shifts compared with those with a more traditional cylindrical cell. As expected from the electrostatic nature of ion trapping in an Orbitrap analyzer (following a formal consideration similar to that in [10]) frequency shifts due to space charge in any of them are substantially $m / z$-independent, which contrasts with strong $m / z$ dependency for FT ICR or RF traps. It has also been discovered that these frequency shifts increase linearly

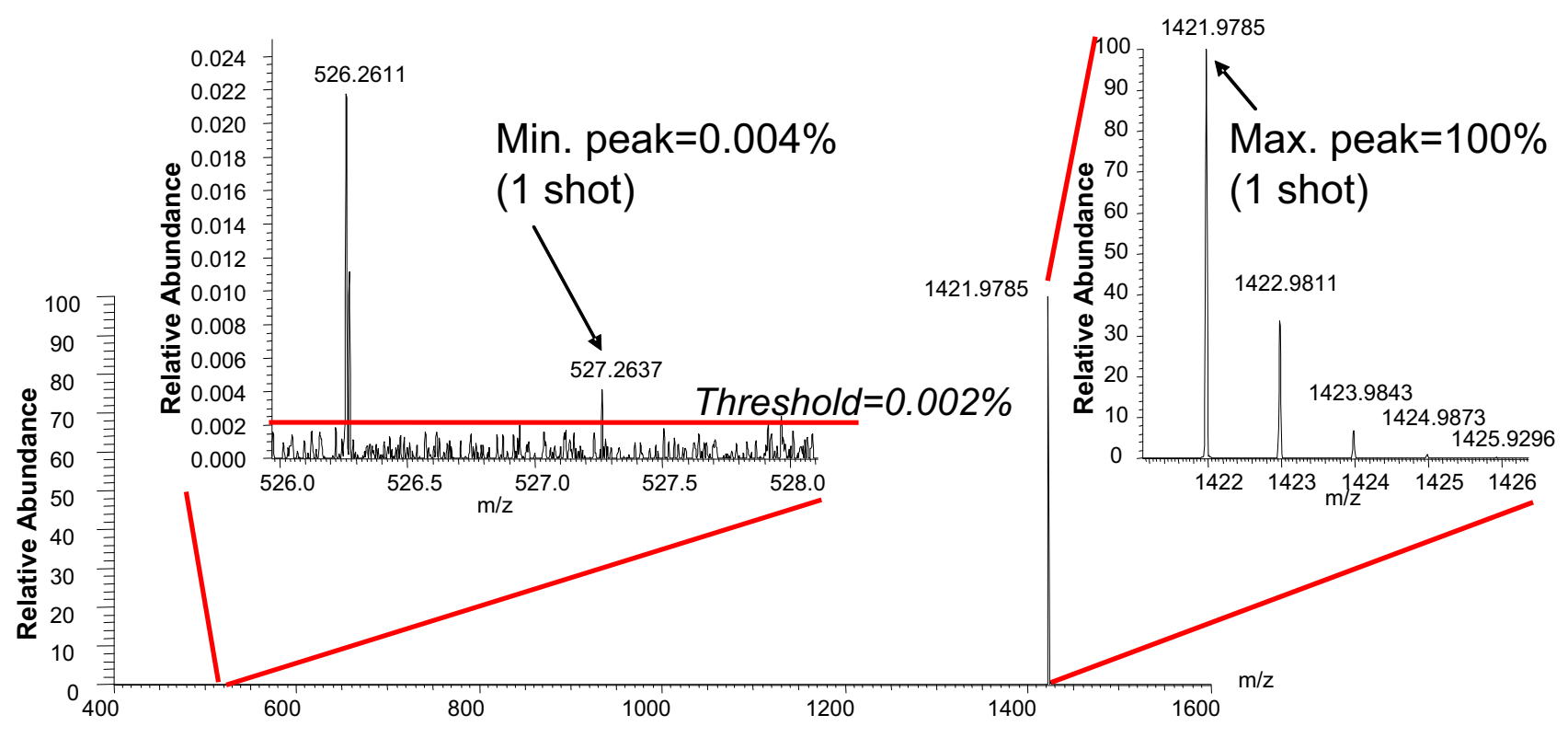

Figure 3. Single full-profile spectrum acquired for $0.76 \mathrm{~s}$ for a $(100 \% / 0.3 \%)$ mixture of Ultramark (monoisotopic peak at $\mathrm{m} / \mathrm{z} 1422$ ) and MRFA (monoisotopic peak at $\mathrm{m} / \mathrm{z} 524$ ) according to method of [2]. Dynamic range of at least 25,000 could be observed for minor isotope of MRFA $\mathrm{m} / \mathrm{z} 524$ at $\mathrm{m} / \mathrm{z} 527$ (its theoretically predicted abundance relative to $\mathrm{m} / \mathrm{z} 524$ is $1.2 \%$, which results in expected intensity of about $0.0035 \%$ relative to Ultramark peak at $\mathrm{m} / \mathrm{z} 1422$ ). 


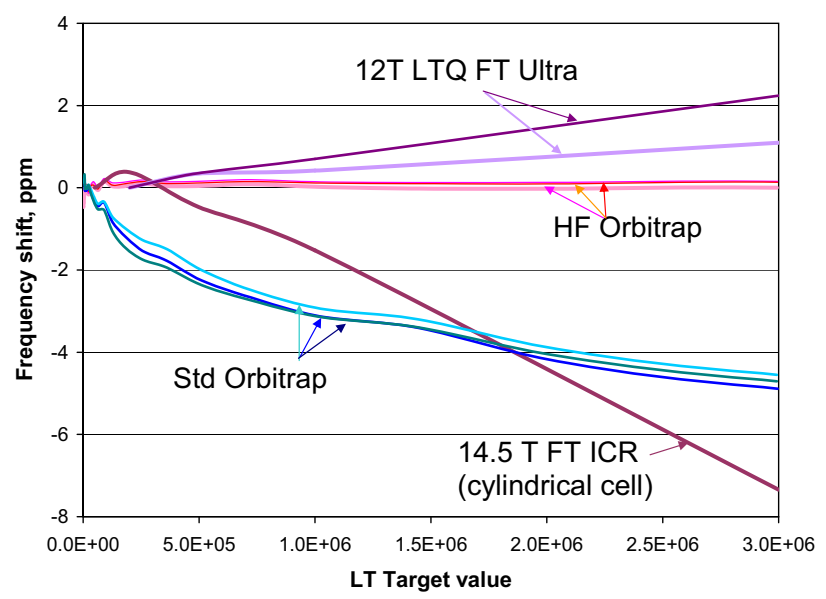

Figure 4. Relative Coulomb frequency shifts in HF and standard Orbitrap, 12T LTQ FT Ultra, and 14.5 T FT ICR in parts per million. Traces correspond to $m / z$ 195, 524, 1422 in both Orbitraps, $m / z$ 524, 1422 in $12 \mathrm{~T}$, and $m / z 524$ in $14.5 \mathrm{~T}$ FT ICR

with the actual ion number (not shown) and even for a standard Orbitrap compare quite favorably with the highest-field FT ICR. Deviation of Orbitrap curves of Figure 4 from linear dependency is linked to the nonlinear relation between LT target value and the actual ion number inside the Orbitrap: at high target values, more ions are lost during the transfer from LT and from the C-trap due to Coulomb expansion of ion clouds in each trap and therefore ion load stops growing at higher target values.

Surprisingly, the graph for HF Orbitrap shows a very small (if any) dependence on space charge even though transmission to HF Orbitrap is higher than that in FT ICR and similar to the standard Orbitrap. This is a direct result of a smaller gap and hence enhanced ion shielding in HF Orbitrap compared with the standard Orbitrap. This shielding effect arises because ions of the same $\mathrm{m} / \mathrm{z}$ spread in rings rotating around the central electrode and bouncing along it, with the central electrode shielding one section of the ring from another section. The closer central electrode comes to the ion ring, the smaller is the proportion of the circumference for Coulomb interaction between ions. In all instruments considered, high mass accuracy during routine measurements is provided by applying an empirical correction to the mass calibration with a linear dependency on ion load and a nonlinear dependency on $\mathrm{m} / \mathrm{z}$. Therefore, these frequency shifts and corresponding mass shifts are normally invisible to the user.

Figure 5 demonstrates that HF Orbitrap is also capable of high resolving power for intact protein analysis once the appropriate base pressure is achieved inside the Orbitrap compartment using higher-temperature bake-out. Resolving power 56,000 for enolase $(46 \mathrm{kDa})$ was observed in a $1.5 \mathrm{~s}$ transient using lowered voltage on the central electrode $(2.5 \mathrm{kV}$ instead of $5 \mathrm{kV}$ in other experiments) at residual pressure $6 \times 10^{-11}$ Torr. Reduction of voltage was required to reduce center-of-mass energy of collision between multiply-charged protein ion and molecules of residual gas. Only then performance of HF Orbitrap analyzer for proteins became similar to that of a standard one which is clearly a negative effect of ion energy increase according to (eq 7).

By careful balancing of construction tolerances and matching electrodes to each other, it is possible to assemble an Orbitrap analyzer with much higher ultimate resolving power than typical for standard instruments. The same is applicable to HF Orbitrap as shown in Figure 6. Ultimate resolving power was obtained using external calibration and $3 \mathrm{~s}$ detection time, which is four times longer comparing to detection time in Figure 2. Isotope clusters with fine structure were resolved with resolving power in excess of 600,000 around $\mathrm{m} / \mathrm{z} 196$, and around $380,000 \mathrm{~m} / \mathrm{z} 525$. As usual for an optimally tuned Orbitrap, this is accompanied by high mass accuracy and correct isotopic ratios within each isotopic envelope. These levels of resolving power are still far below, and will remain below record values obtained in FT ICR, but it is more than adequate even for most demanding complex mixtures such as petroleum or humic acids.

\section{Conclusions}

A new, high-field Orbitrap analyzer indeed provides the expected improvement of resolving power per unit time. Through careful balancing of construction tolerances and operation parameters, HF Orbitrap can provide maximum resolving power in excess of 350,000 at $\mathrm{m} / \mathrm{z} 524$ and 600,000 at $\mathrm{m} / \mathrm{z} 195$ isotopic resolution of proteins above 40 $\mathrm{kDa}$ and a single-shot dynamic range of 25,000. It was also found that mass shifts in HF Orbitrap depend very little on space charge inside the analyzer.

At the same time, HF Orbitrap analyzer appeared to be more demanding than a standard one with regards to the alignment of ion optics, residual pressure, and variations of tuning parameters. Further research and development is required to provide a robust infrastruc-

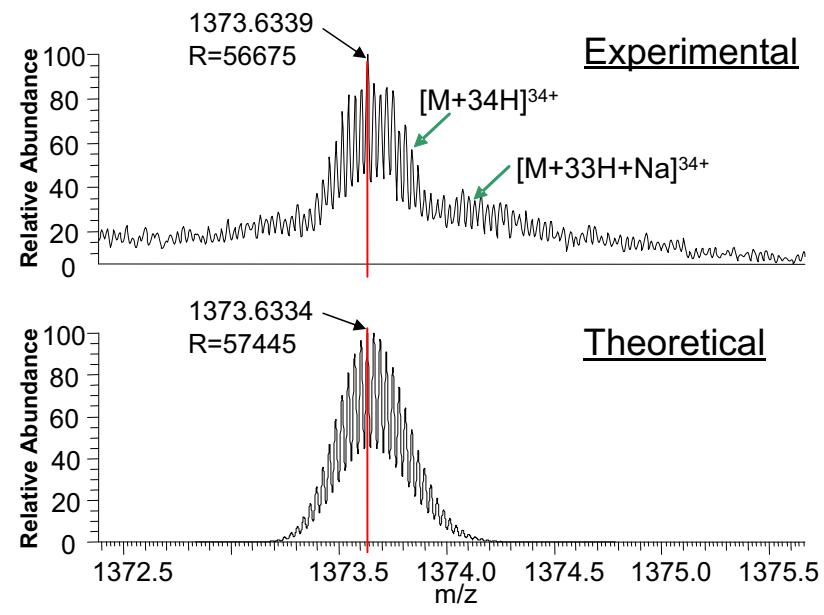

Figure 5. Spectrum of yeast enolase 1 (molecular weight $46 \mathrm{kDa}$ ), charge state $34+$ with external calibration. 


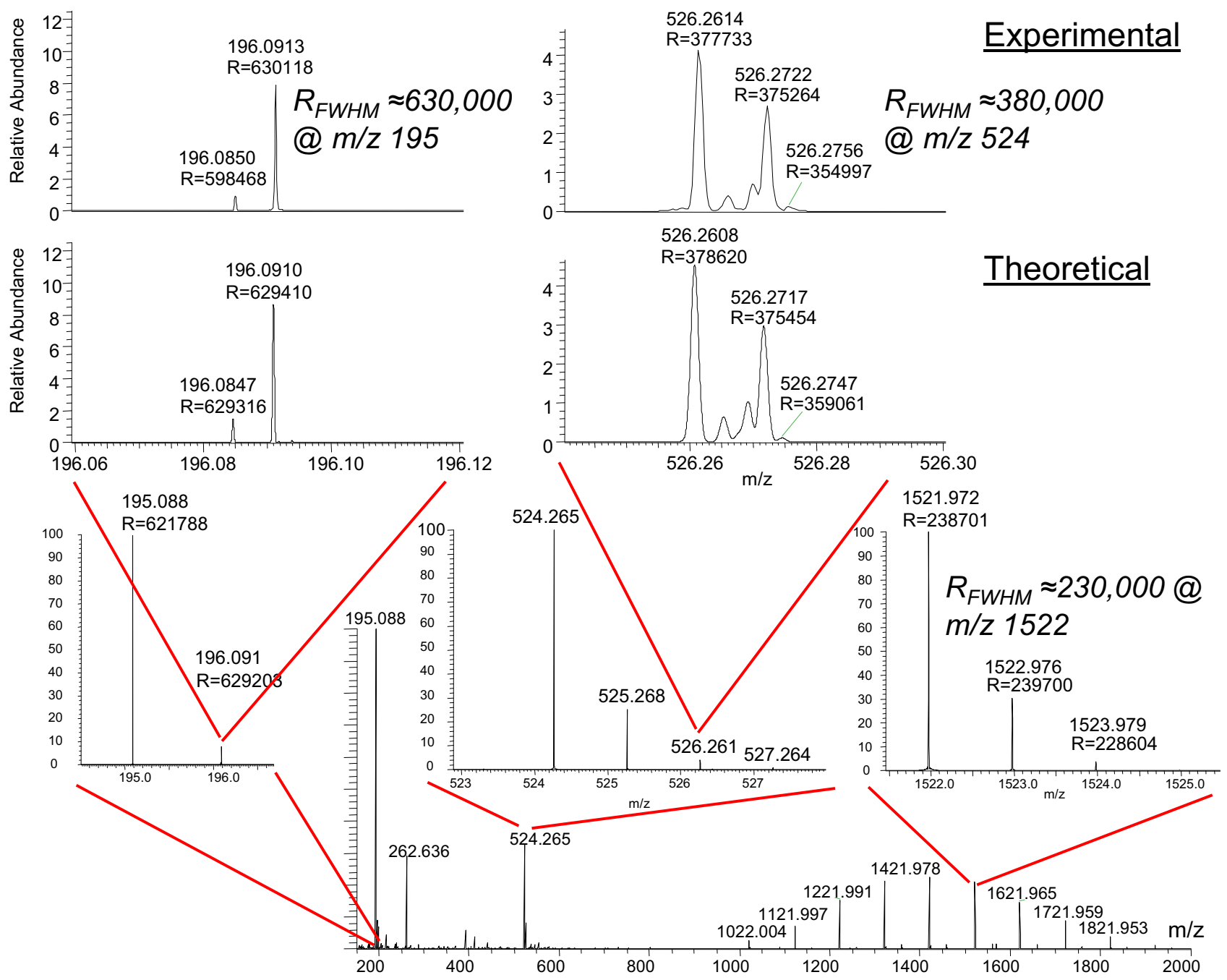

Figure 6. Ultimate resolving power obtained using $3 \mathrm{~s}$ detection time (four times longer comparing to detection time in Figure 2) and EXTERNAL calibration. Experimental values are presented on panels for corresponding $\mathrm{m} / \mathrm{z}$. Isotope clusters with fine structure are presented in insets along with corresponding theoretical patterns normalized to monoisotopic peak.

ture and automatic procedures that would allow this analyzer to be used in serial instruments.

\section{Acknowledgments}

The authors thank Dr. Christopher Hendrickson from the National High Magnetic Field Laboratory for providing the 14.5 T FT ICR instrument for space charge shift measurements, and Dr. Mike Senko for carrying out measurements. They also thank Professor Neil Kelleher for providing data from 12 T LTQ FT Ultra instrument. The authors are grateful to Wilko Balschun for mechanical design of HF Orbitrap construction, Alexander Kholomeev for developing electronics for experiments, Dr. Jens Griep-Raming and Dr. Stevan Horning for support and fruitful discussions. The research leading to these results has received funding from the European Commission's 7th Framework Programme (grant agreement HEALTH-F4-2008-201648/PROSPECTS).

\section{References}

1. Marshall, A. G.; Guan, S. Advantages of High Magnetic Field for Fourier Transform Ion Cyclotron Resonance Mass Spectrometry. Rapid Commun. Mass Spectrom. 1996, 10(14), 1819-1823.
2. Marshall, A. G.; Hendrickson, C. L.; Jackson, G. S. Fourier Transform Ion Cyclotron Resonance Mass Spectrometry: A Primer. Mass Spectrom. Rev. 1998, 17, 1-35.

3. Makarov, A. Electrostatic Axially Harmonic Orbital Trapping: A HighPerformance Technique of Mass Analysis. Anal. Chem. 2000, 72, $1156-$ 1162.

4. Makarov, A.; Denisov, E.; Kholomeev, A.; Balschun, W.; Lange, O.; Horning, S.; Strupat, K. Performance Evaluation of a Hybrid Linear Ion Trap/Orbitrap Mass Spectrometer. Anal. Chem. 2006, 78, 2113-2120.

5. Wenger, C. D.; Boyne, M. T.; Ferguson, J. T.; Robinson, D. E.; Kelleher, N. L. Versatile Online-Offline Engine for Automated Acquisition of High-Resolution Tandem Mass Spectra. Anal. Chem. 2008, 80, 80558063.

6. Schaub, T. M.; Hendrickson, C. L.; Horning, S.; Quinn, J. P.; Senko, M. W.; Marshall, A. G. High-Performance Mass Spectrometry: Fourier Transform Ion Cyclotron Resonance at 14.5 Tesla. Anal. Chem. 2008, 80, 3985-3990.

7. Schwartz, J. C.; Senko, M. W.; Syka, J. E. P. A Two-Dimensional Quadrupole Ion Trap Mass Spectrometer. J. Am. Soc. Mass Spectrom. 2002, 13, 659-669.

8. Makarov, A.; Denisov, E.; Lange, O.; Horning, S. Dynamic Range of Mass Accuracy in LTQ Orbitrap Hybrid Mass Spectrometer. J. Am. Soc. Mass Spectrom. 2006, 17, 977-982.

9. Wieghaus A., Froehlich U., Malek R., Horning S. The Grid Cell: A New Cell Design for Reduced Z-Axis Ejection in Fourier Transform Ion Cyclotron Mass Spectrometry. Proceedings of the 54th ASMS Conference; Seattle, WA, May, 2006.

10. Jeffries, J. B.; Barlow, S. E.; Dunn, G. H. Theory of Space-Charge Shifts of Ion Cyclotron Resonance Frequencies. Int. J. Mass Spectrom Ion Processes 1983, 54, 169-187. 